\title{
Systemkombinierende untertägige Pumpspeicherkraftwerke für eine integrative und effiziente Energietransformation
}

\author{
Franz Georg Pikl ${ }^{1}$ Wolfgang Richter ${ }^{1} \cdot$ Gerald Zenz ${ }^{1}$ \\ Online publiziert: 13. März 2020 \\ ○ Der/die Autor(en) 2020
}

\section{Zusammenfassung}

Pumpspeicherkraftwerke sind ein effizientes und langlebiges Nonplusultra für ausgeglichene und stabile elektrische Energiesysteme. Unterirdische Pumpspeicherkraftwerke werden zum vielversprechenden Ansatz zur Bewältigung verschiedener Herausforderungen und ermöglichen zudem vollkommen neue und in mehrfacher Weise einsetzbare Anlagenkonzepte. Thermische Pumpspeicherkraftwerke als mechanisch und thermisch kombinierte Energiespeicher bilden die innovative technologische Evolution für eine universelle und ressourceneffiziente, regenerative Energieversorgung.

\section{Integrative and future-proof pumped-storage hydropower}

\begin{abstract}
Pumped-storage hydropower plants are the efficient and most mature energy storage for balanced and stable electrical energy systems. Underground pumped hydro is becoming a promising concept to address various challenges and enabling completely new, multifunctional and synergetic plant concepts regardless topography. Underground seawater pumpedstorage, with the ocean as an infinite upper reservoir and the upgrade of existing reservoirs by deep underground caverns utilize existing resources for an even more economical realization of pumped hydro. Thermal pumped-storage power schemes as electrically and thermally combined energy storage are the innovative technological evolution for a holistic and resource-efficient, regenerative energy supply.
\end{abstract}

\section{Hydraulische Energiespeicher- technologien}

Seit Beginn des 20. Jahrhunderts sind Pumpspeicherkraftwerke (PSKW) unabdingbares und zuverlässiges Rückgrat des elektrischen Energiesystems. Aufbauend auf dem Prinzip der bewährten hydroelektrischen Speichertechnologie werden PSKW durch einen interdisziplinären Entwicklungsprozess seit jeher an die Bedürfnisse der Elektrizitätswirtschaft angepasst, wodurch sie mit hochflexiblen

Dieser Beitrag ist ursprünglich erschienen in WasserWirtschaft 109 (2019) [Ausgabe 9].

Franz Georg Pikl

franz.pik1@ tugraz.at

Wolfgang Richter

wolfgang.richter@tugraz.at

Gerald Zenz

gerald.zenz@tugraz.at

1 Institut für Wasserbau und Wasserwirtschaft, Technische Universität Graz, Stremayrgasse 10/2, 8010 Graz, Österreich
Betriebsweisen auch den Anforderungen einer zusehends erneuerbar basierten, volatilen Primärenergieerzeugung gerecht werden. Mit der notwendigen Ökologisierung und Dekarbonisierung des Energiesystems, der Industrie und des Verkehrs steigt zudem der Bedarf an jederzeit verfügbarer, effizient gespeicherter Energie und Leistung, unabhängig von der temporär entkoppelten Erzeugung unerschöpflicher aber intermittierender Energieträger.

Energiespeicher sind auch am thermischen Sektor von zentraler Bedeutung, um den klima- und umweltgerechten Energieumbau zu ermöglichen. Unter anderem sind großtechnische saisonale Wärmespeicher die forcierte Technologie für eine versorgungssichere und bedarfsgerechte $\mathrm{Be}$ reitstellung von ökologischer Fernwärme für urbane Gebiete und Städte. Auch hier spielt das natürliche Speichermedium Wasser aufgrund dessen idealer thermischer Eigenschaften eine entscheidende Rolle. Hocheffizient ist dabei die seit Jahrzehnten erprobte Heißwasser-Wärmespeicherung in unausgekleideten Felskavernen mit saisonalen Wirkungsgraden von über $90 \%$ (Åsberg 2011).

Es ist naheliegend, die Vorteile beider etablierter hydraulischer Energiespeichertechnologien durch die energetische 
Doppelnutzung des Wassers in sogenannten thermischen PSKW zu vereinen (Pikl 2017). Zusammen mit physikalischer Vernetzung von elektrischer und thermischer Energieinfrastruktur erlaubt diese technologische Errungenschaft die effiziente und sektorenübergreifende Kopplung der verschiedenen erneuerbaren Primärenergieerzeuger mit diversen Energiekonsumenten zur nachhaltigen und sektorenübergreifenden Energieversorgung.

\section{Untertägige Pumpspeicherkraftwerke}

Bereits für die ersten Hochkulturen der Menschheitsgeschichte waren insbesondere zur Trinkwasserversorgung errichtete Untertagebauwerke unverzichtbare Grundlage für Wohlstand, Wachstum und sozialen Frieden. Die beständigen und robusten, bei stabilen Untergrundverhältnissen jedenfalls bis zur nächsten Gebirgsfaltung dauerhaften Bauwerke, weisen zudem technische Lebensdauern auf, die in der Regel die Nutzungsdauern übersteigen (Garbrecht 1995).

Auch für PSKW liegt die Zukunft unter Tage. Denn der zentrale Eckpfeiler der entwickelten Energiespeicherkombination ist die vollkommen unterirdische Errichtung von PSKW mit geschlossenem Wasserkreislauf (Abb. 1).

Die Unabhängigkeit gegenüber topographischen und geographischen Gegebenheiten ermöglicht nicht nur die standortvariable Errichtung in vorerkundeten, geeigneten geologischen Bereichen in Tallagen bzw. in der peripheren Umgebung von Ballungsräumen und Städten, sondern erleichtert auch die Erschließung und die Logistik für Bau und Betrieb. Die Nähe zu bestehenden Energienetzen stellt eine wirtschaftliche Anbindung an das Energiesystem sicher, wobei unter Umständen eine direkte Integration ins Niederspannungsnetz eine Option darstellt.

Durch die übereinanderliegende Anordnung der Speicherkavernen ermöglicht ein kurzes, primär auf den Druckschacht als direkte vertikale Verbindung reduziertes Triebwassersystem eine kompakte Ausführung (Pikl 2017). Mit der Realisierung von sehr großen und von natürlichen Gegebenheiten unabhängigen Fallhöhen können auszubrechende Speichervolumina einerseits und die Dimensionen der Triebwasserführung und der Maschinen andererseits für festgelegte Energiemengen bzw. installierte Kraftwerksleistungen signifikant reduziert werden. Dadurch werden Investitionen und die Wirtschaftlichkeit eines Projekts direkt optimierbar. Insbesondere die mehrstufige Ausführung (Abb. 1) ermöglicht die Skalierbarkeit von PSKW in die Tiefe, so dass mit der seriellen Stufenteilung, neben der üblichen Parallelschaltung von Maschinensätzen in den Kraftstationen, auch erhöhte flexible Betriebsweisen der Gesamtanlage einhergehen.
Außerdem wird maximale Umwelt- und Naturverträglichkeit über den gesamten Lebenszyklus gewährleistet. Wertvolle Flächenressourcen bleiben unberührt, mit dem künstlich geschaffenen Wasserkörper wird weder in den natürlichen Wasserhaushalt sowohl von Grund- als auch Oberflächenwässern eingegriffen, noch spielen Schwallund Sunkerscheinungen als gewässerökologische Beeinträchtigungen eine Rolle. Der geschlossene, von natürlichen Gewässern und der obertägigen Biosphäre entkoppelte unterirdische Wasserkreislauf schließt den Sedimenteintrag, Verlandungen und Verdunstungsverluste grundsätzlich aus und sichert gleichbleibendes Wasservolumen, sowie uneingeschränkten Betrieb über die gesamte Lebensdauer. Der Kraftwerksbetrieb ist außerdem frei von Emissionen jeglicher Art. Auch wird das Landschaftsbild nicht beeinflusst.

\section{Thermische Pumpspeicherkraftwerke}

Basierend auf der unterirdischen Errichtung werden die energetisch hybrid genutzten PSKW (Abb. 2) technisch an die bis $\mathrm{zu} 90^{\circ} \mathrm{C}$ heißen Wassertemperaturen und dadurch bedingten thermischen bzw. mechanischen Beanspruchungen adaptiert (Pikl 2017; Pikl et al. 2017).

Das hohe Temperaturniveau wird mit der Vernetzung von verschiedenen Wärmequellen und der effizienten Wärmepumpentechnologie über Fernwärmeübertragungsleitungen erreicht. Das Ein- und Ausspeichern der Wärme in den Wasserkörper des PSKW erfolgt mit Wärmetauschern bzw. Sekundärsystemen.

Das umgebende Gebirge der heißwasserbeaufschlagten Bauwerke ist nicht nur natürliche Wärmedämmung und schützt vor äußeren Einflüssen, sondern ist auch zusätzliche wärmespeichernde Masse. Darüber hinaus stellt der Luftdruckausgleichsschacht konstanten Luftdruck im Speicherkavernensystem unabhängig von den Staupegeln sicher und verhindert Wärme- und Verdunstungsverluste an die Umwelt (Pikl 2017). Der Luftstrom ausgleichender Luftmassen kann überdies mit einer bidirektional anströmbaren Luftturbine energetisch genutzt werden.

Untertägige Heißwasser-PSKW mit geschlossenem Wasserkreislauf ermöglichen außerdem die thermische Nutzung von hydraulischen Strömungsverlusten des Kraftwerkbetriebs (Pikl et al. 2019). Die größtenteils in Wärme übergehenden Verluste werden ohne weitere Betriebseinrichtungen zur direkt verwertbaren thermischen Energie, wodurch der Gesamtwirkungsgrad des Heißwasser-Pumpspeichers auf über $98 \%$ in Bezug auf die elektrische Energie gesteigert werden kann. Darüber hinaus bietet die jederzeitige Erweiterbarkeit von Speichervolumen bzw. die mögliche asymmetrische Größenauslegung der Speicherkavernen oder ein Leistungsausbau zusätzliche Flexibilisierungsoptionen, um das kombinierte Speichersystem sich verändern- 


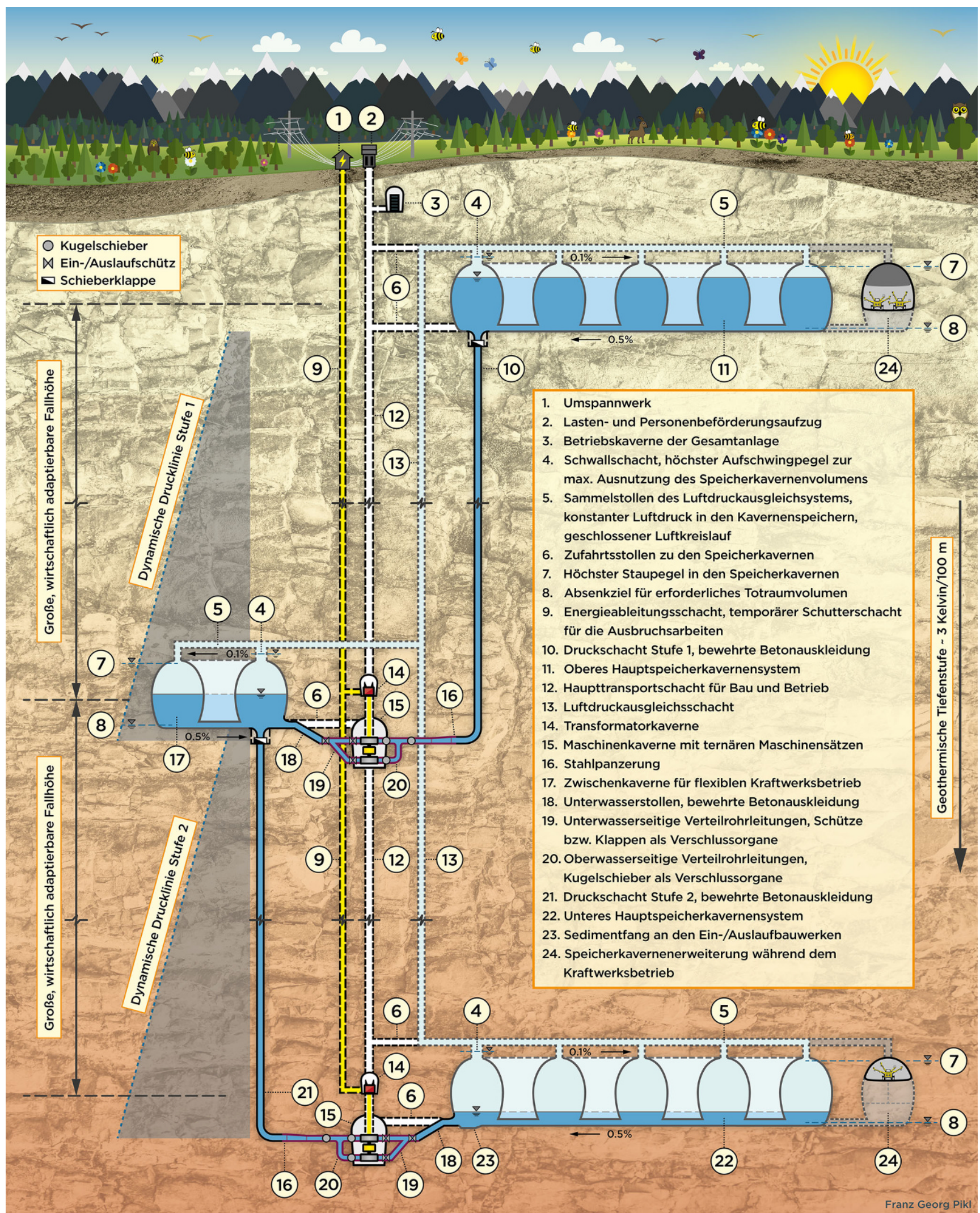

Abb. 1 Zweistufige, vollständig unterirdische PSKW-Anlage entwickelt für große Fallhöhen und hochflexiblen Kraftwerksbetrieb 


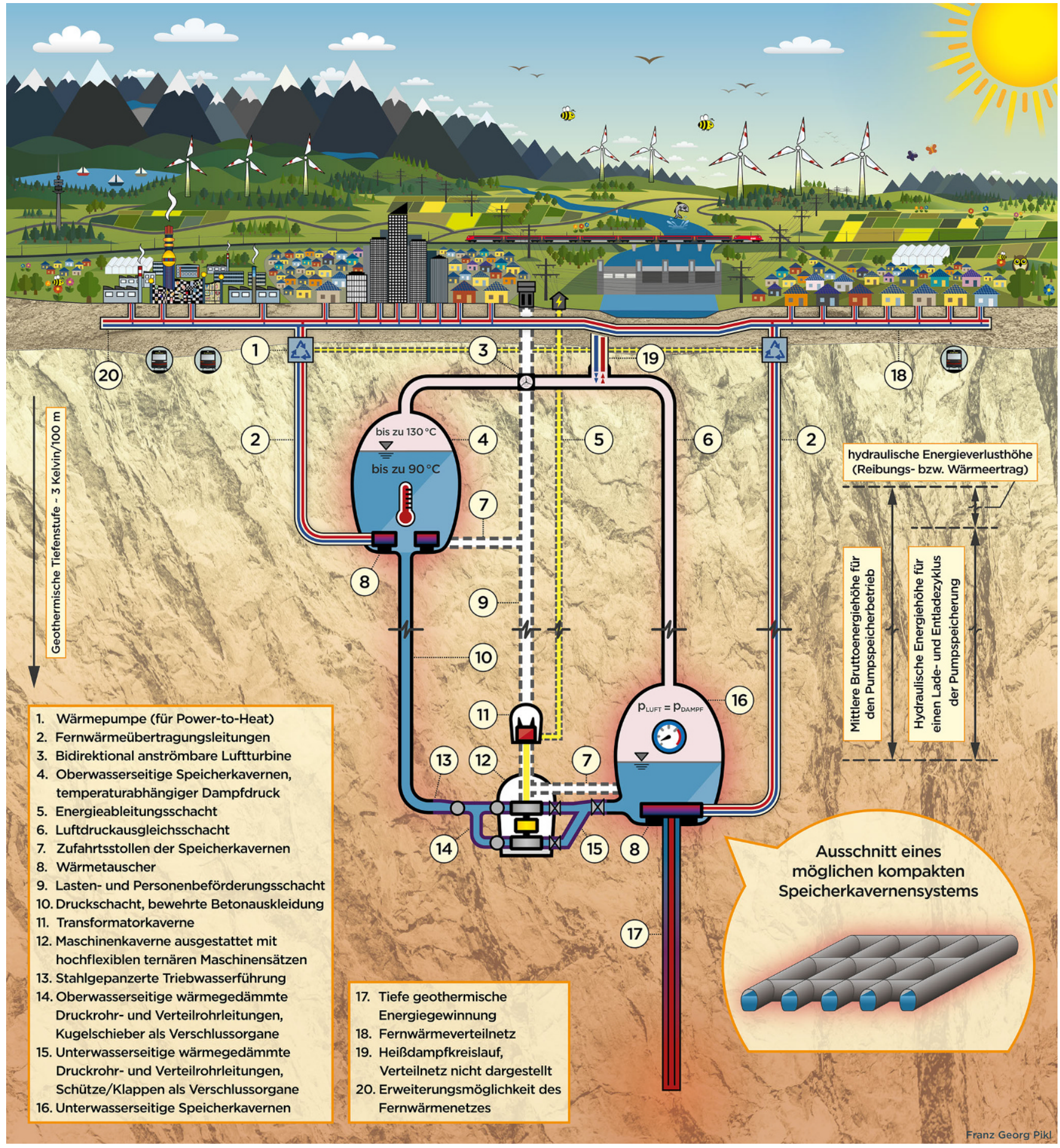

Abb. 2 Vision eines großtechnischen thermischen PSKW zur universellen und effizienten Energieversorgung ganzer Städte und deren peripheren Umgebung

den Versorgungsanforderungen oder (energie-) wirtschaftlichen Randbedingungen anzupassen.

Die Wärmespeicherung erfolgt nicht nur im Wasserkörper und in den umgebenden Gebirgsbereichen der Speicherkavernen, sondern auch im verbleibenden Hohlraumvolumen des Speicherkavernensystems (Pikl et al.
2019). Bereits hochtemperiertes Wasser wird dabei aus dem Pumpspeichersystem entnommen, um daraus mit energieeffizienten Technologien, insbesondere Hochtemperatur-Wärmepumpen, Wasserdampf zu erzeugen, der anschließend im Hohlraumvolumen der unterirdischen Speicherbauwerke, unabhängig von den anderen Energie- 


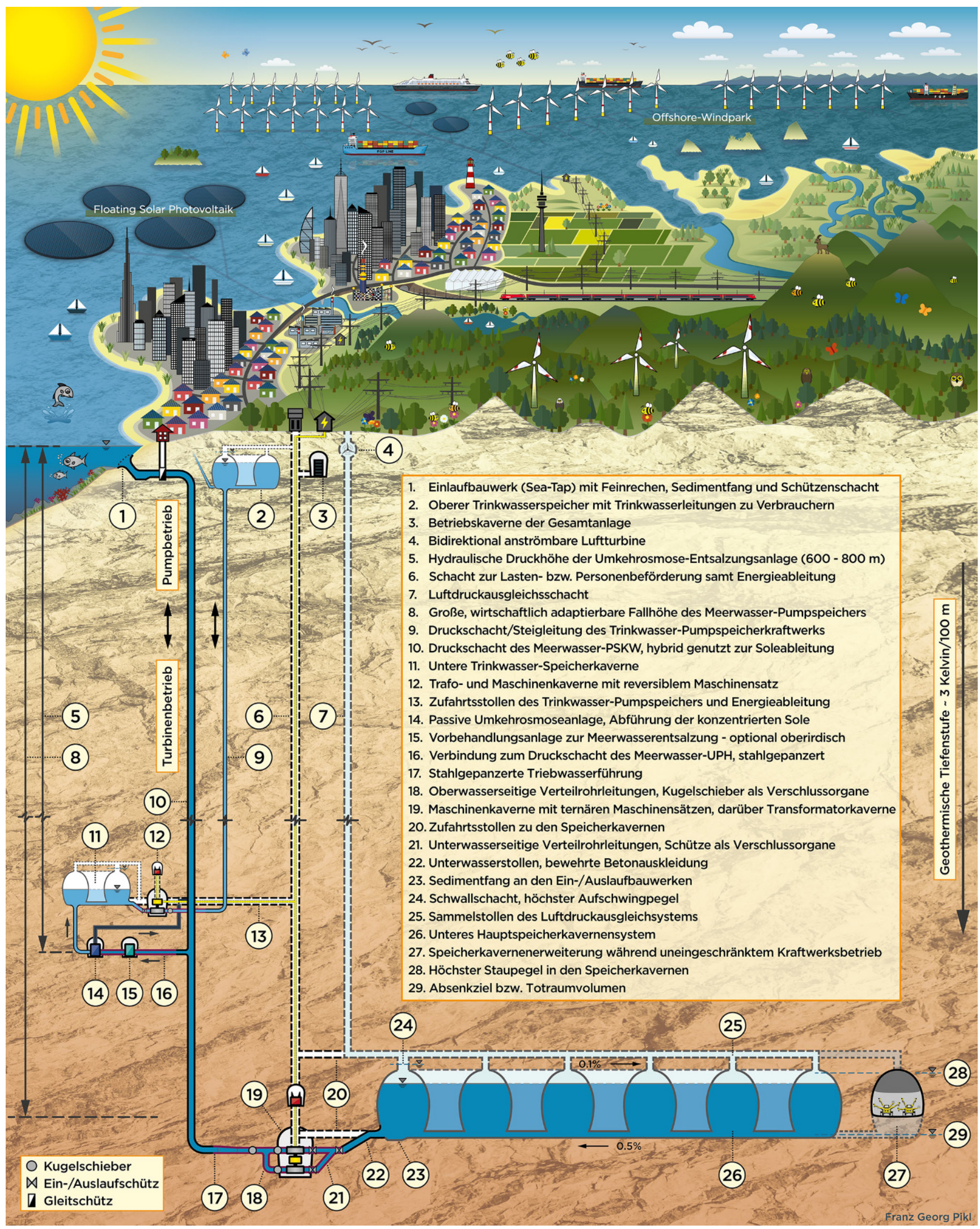

Abb. 3 Unterirdisches Meerwasser-PSKW, synergetisch kombiniert mit einer energieeffizienten passiven Umkehrosmose-Meerwasserentsalzungsanlage bzw. der optionalen Ausführung eines Süßwasser-PSKW 


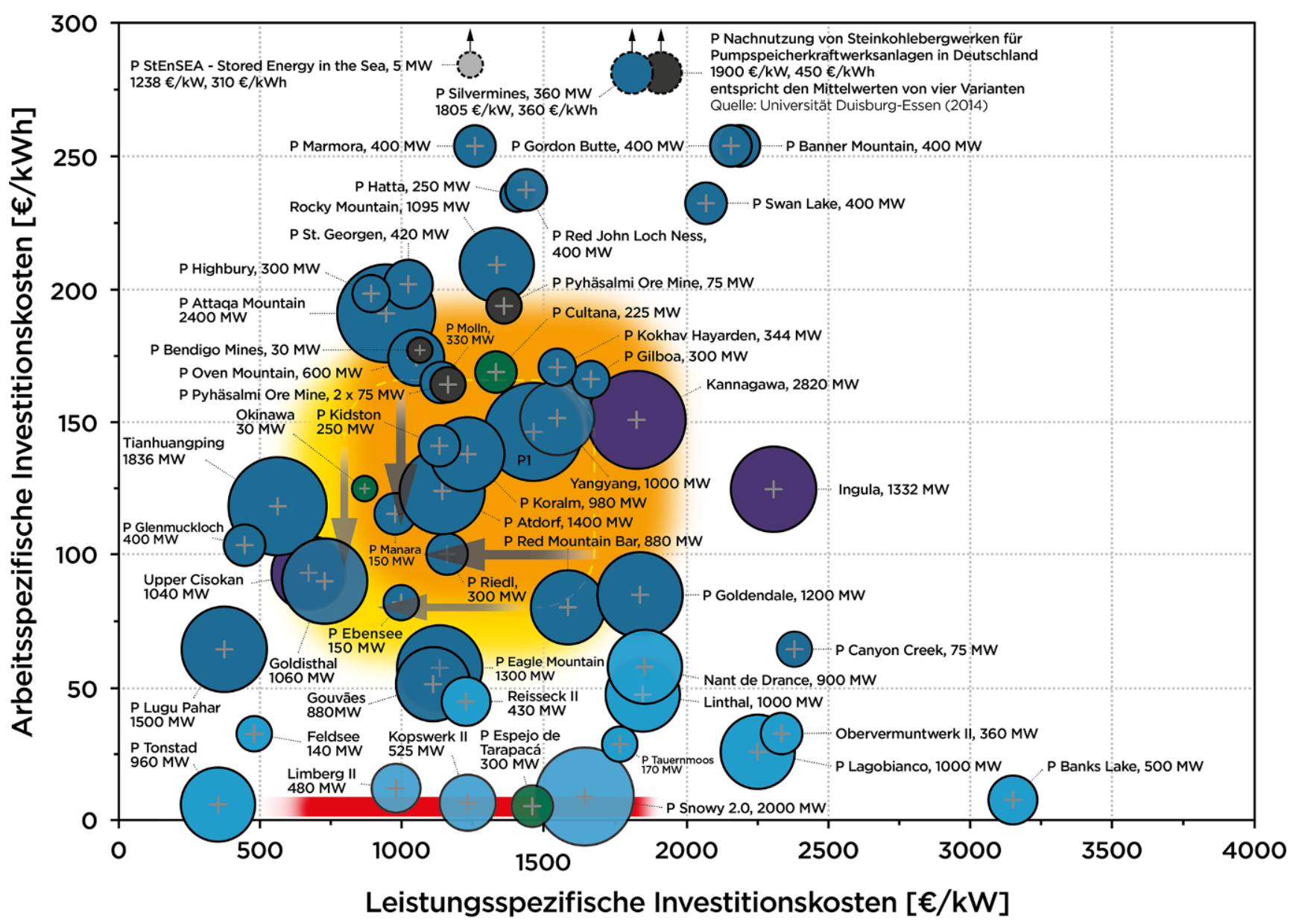

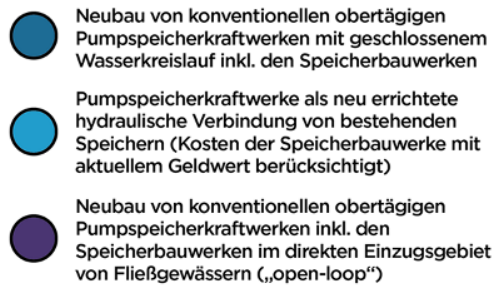
von Fließgewässern (open-loop“)

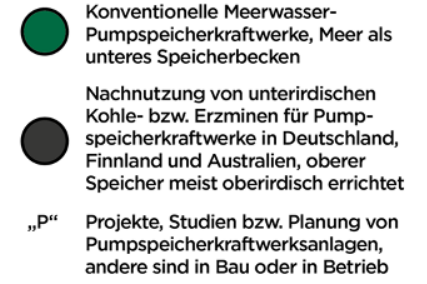

P1 P Navajo Energy Storage Station, $2210 \mathrm{MW}$

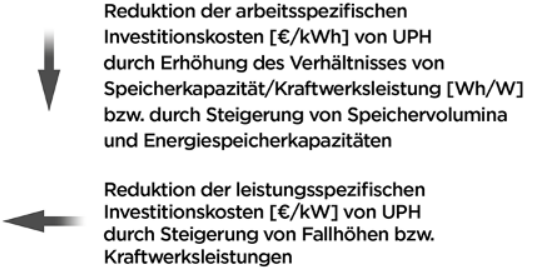

Unterirdische Pumpspeicherkraftwerke

Neubau von vollkommen unterirdischen Pumpspeicherkraftwerken (UPH) inkl. den Speicherkavernen, Bereich spezifischer Investitionskosten

Nutzung von bestehenden oberirdischen Speichern in Kombination mit neu errichteten, tief gelegenen Speicherkavernen für unterirdische Pumpspeicherkraftwerke (U-UPH), Bereich spezifischer Investitionskosten

Thermische Pumpspeicherkraftwerke: Neubau von unterirdischen Pumpspeicherkraftwerken kombiniert mit thermischer Energiespeicherung (THERMAL-UPH), Bereich spezifischer Investitionskosten

Abb. 4 Gegenüberstellung der spezifischen Investitionen von PSKW mit leistungsspezifischen $[€ / \mathrm{kW}]$ und arbeitsspezifischen Kosten [€/kWh], bezogen auf die installierte Turbinenleistung und den nutzbaren Energieinhalt des einmalig vollgefüllten Oberbeckens, Preisbasis 2019

speichermöglichkeiten, vorgehalten wird. Aufgrund des energieintensiven Phasenüberganges können beträchtliche zusätzliche thermische Energiemengen in der Gasphase gespeichert werden, die über einen geschlossenen Kreislauf sowohl für Industrieprozesse als auch für das regenerative Beheizen von Gewächshäusern zur lokalen und urbanen, jahreszeitlich unabhängigen Nahrungsmittelproduktion eingesetzt werden können.
In Kaltwasser-PSKW, dem Pendant zur Heißwasser-Variante, wird der Wasserkörper mit Kältemaschinen auf etwa $0{ }^{\circ} \mathrm{C}$ gekühlt. Über Fernkälteübertragungsleitungen kann in entsprechenden klimatischen Gebieten großtechnisch Kälte zur Raumkühlung bereitgestellt werden. Die Kombination von Pumpspeichern mit Wärme- und Kältesystemen ermöglicht eine maßgeschneiderte und flexible Anpassung an die 
jeweiligen geographischen und versorgungstechnischen Bedürfnisse.

Langlebige unterirdische PSKW erfahren durch die innovative Kombination mit thermischer Energiespeicherung bei nahezu unverändertem Ressourcenverbrauch gegenüber konventionellen PSKW durch den markant gesteigerten Energieumsatz einen bedeutenden gesamtökonomischen Wertzuwachs. Die großtechnische und umweltfreundliche Speichertechnologie ermöglicht Synergien und bis dato nicht da gewesene Potenziale durch die gesamtsystematische Interoperabilität des Energiesystems und die Vereinigung etablierter Technologien wirtschaftlich und effizient nutzbar zu machen.

\section{Unterirdische Meerwasser- Pumpspeicherkraftwerke}

Konventionelle Meerwasser-PSKW, die sich dem Meer als unterem Reservoir bedienen, sind vermehrt projektierte Alternative zu herkömmlichen Süßwasser-Pumpspeichern, jedoch vom Relief der Erdkruste küstennaher Gegenden abhängig. Bildet umgekehrt der Ozean das natürliche Oberbecken und tiefliegende Speicherkavernen das künstlich geschaffene Unterbecken, können nicht nur die genannten Vorzüge der unterirdischen Bauweise genutzt werden, sondern leistungsstarke PSKW in unmittelbarerer Nähe von Küstenmetropolen und großen Verbrauchern realisiert werden (Abb. 3).

Wohlstandssichernde Lebensgrundlage ist neben der zuverlässigen Energieversorgung auch die Bereitstellung ausreichenden und sauberen Trinkwassers. In zum Teil wasserarmen Küstenregionen wird die essenzielle Wasserversorgung oftmals durch Meerwasserentsalzungsanlagen ergänzt. Die bedeutendste der allesamt energieintensiven Entsalzungsverfahren ist die Umkehrosmose, bei der Meerwasser unter hohem Druck durch semipermeable Membranen gepresst wird.

Diese Meerwasserentsalzungsanlagen können synergetisch und energieeffizient mit unterirdischen MeerwasserPSKW zusammengeführt werden. Für die Umkehrosmose wird der erforderliche Druck dabei nicht klassisch mit Hochdruckpumpen erzeugt, sondern über den hybrid genutzten Druckschacht des Meerwasser-PSKW und den in entsprechenden Tiefenlagen anstehenden, immer verfügbaren hydraulischen Druck. Das saubere Süßwasser wird entweder in eigens dafür vorgesehenen Kavernen zwischengespeichert oder direkt zu Verbrauchern transportiert. Das Hochpumpen des sauberen Trinkwassers erlaubt die direkte Integration in den Speicherbetrieb des Pumpspeichersystems. Die zusätzliche Errichtung oberer Speicherkavernen ermöglicht außerdem die Realisierung eines vollständig vom Meerwasser-PSKW unabhängigen Süßwasser-Pumpspeichersystems.

\section{Wirtschaftlichkeit unterirdischer Pumpspeicherkraftwerke}

Ressourceneffiziente PSKW weisen bei hochwertiger Auslegung, regelmäßiger Wartung und konsequenten Instandhaltungsmaßnahmen technisch unbegrenzte Lebensdauern auf. Sie sind systemrelevante Vermögenswerte eines sich stets wandelnden Energiesystems mit steigendem Energiebedarf. Untersuchungen zeigen, dass vollständig unter Tage errichtete PSKW nicht nur sehr kosteneffizient realisiert werden können, sondern zumeist auch wirtschaftlicher sind als neue, konventionelle PSKW mit oberirdschen Speichern (Pikl et al. 2019; Pikl und Richter 2019; Chas. T. Main, Inc. 1982; Willett 1981), (Abb. 4).

Ausschlaggebend dafür ist, dass bei vorgegebenen Investitionsvolumina durch große, adaptierbare hydraulische Höhen gezielt wirtschaftlich maßgeschneiderte Anlagenkonzepte ausgearbeitet werden können. Eine besonders ökonomische Möglichkeit bietet zudem die Erweiterung von bestehenden, obertägigen Speichern durch tiefliegende Speicherkavernen, um eine signifikante Erhöhung des Energieinhalts herbeizuführen und dabei ähnlich den unterirdischen Meerwasser-PSKW bereits vorhandene Speicherpotenziale nachhaltig zu nutzen (Abb. 4). Die gesamtsystematische und interdisziplinäre Vereinigung von etablierten Technologien ermöglicht darüber hinaus Synergien und die Erschließung neuer ökonomischer Potenziale. Eine ausgefeilte kombinierte Nutzung verfügbarer und beständiger Technologien kann regenerative Energien für verschiedenste Anwendungszwecke höchst effizient aus einem vereinten hydraulischen Energiespeichersystem zur Verfügung stellen.

Funding Open access funding provided by Graz University of Technology.

Open Access Dieser Artikel wird unter der Creative Commons Namensnennung 4.0 International Lizenz veröffentlicht, welche die Nutzung, Vervielfältigung, Bearbeitung, Verbreitung und Wiedergabe in jeglichem Medium und Format erlaubt, sofern Sie den/die ursprünglichen Autor(en) und die Quelle ordnungsgemäß nennen, einen Link zur Creative Commons Lizenz beifügen und angeben, ob Änderungen vorgenommen wurden.

Die in diesem Artikel enthaltenen Bilder und sonstiges Drittmaterial unterliegen ebenfalls der genannten Creative Commons Lizenz, sofern sich aus der Abbildungslegende nichts anderes ergibt. Sofern das betreffende Material nicht unter der genannten Creative Commons Lizenz steht und die betreffende Handlung nicht nach gesetzlichen Vorschriften erlaubt ist, ist für die oben aufgeführten Weiterverwendungen des Materials die Einwilligung des jeweiligen Rechteinhabers einzuholen.

Weitere Details zur Lizenz entnehmen Sie bitte der Lizenzinformation auf http://creativecommons.org/licenses/by/4.0/deed.de. 


\section{Literatur}

Åsberg C (2011) Solvärme med säsongslager i Lyckebo - Utredning avvärmeförluster och dimensionering av solfält. Universitet, Uppsala

Chas T (1982) Main. Inc : (Report on Technical Feasibility of Underground Pumped Hydroelectric Storage in a Marble Quarry Site in the Northeast United States. Washington: U.S. Department of Energy)

Garbrecht G (1995) Meisterwerke antiker Hydrotechnik. Teubner, Stuttgart, Leipzig

Pikl FG (2017) Kombination der Pumpspeichertechnologie mit thermischer Energiespeicherung. Masterarbeit. Technische Universität Graz, Graz

Pikl FG, Richter W, Zenz G (2017) Pumped storage technology combined with thermal energy storage-Power station and pressure tunnel concept. Geomech Tunn 10(5):611-619
Pikl FG, Richter W, Zenz G (2019) Großtechnische, wirtschaftliche und effiziente untertägige Energiespeicher. Geomech Tunn 12(3):251-269

Pikl FG, Richter W (2019) Economic analysis of pumped-storage hydropower facilities. Unpublished (Research study)

Universität Duisburg-Essen (Hrsg) (2014) Entwicklung eines Realisierungskonzeptes für die Nutzung von Anlagen des Steinkohlebergbaus als unterirdische Pumpspeicherkraftwerke - Zusammenfassender Abschlussbericht. Essen

Willett DC (1981) Preliminary design study of underground pumped hydro and compressed-air energy storage in hard rock. Vol. 1: Executive Summary, Technical planning study EM-1589 prepared by Acres American. Buffalo 